\title{
On the Internal Consistency of Short-Term, Medium-Term, and Lon-Term Oil Price Forecasts
}

Christian Pierdzioch

Saarland Universität 66041 Saarbrücken

Georg Stadtmann

Europa Universität Viadrina 15207 Frankfurt/Oder
Jan-Christoph Rülke

WHU-Otto Beisheim School of Management jan-c.ruelke@whu.edu

WHU - Working Paper

Series in Economics

WP 11/02 


\title{
On the Internal Consistency of Short-Term, Medium-Term, and Long-Term Oil Price Forecasts
}

\author{
Christian Pierdzioch $^{a}$, Jan Christoph Rülke ${ }^{b, *}$ and Georg Stadtmann ${ }^{c}$
}

February 2011

\begin{abstract}
We derive internal consistency restrictions on short-term, mediumterm, and long-term oil price forecasts. We then analyze whether oil price forecasts extracted from the Survey of Professional Forecasters conducted by the European Central Bank satisfy these internal consistency restrictions. We find that neither short-term forecasts are consistent with medium-term forecasts nor that medium-term forecasts are consistent with long-term forecasts. Using a more complex expectation formation structure featuring a distributed lag structure, however, we find stronger evidence of internal consistency of mediumterm forecasts with long-term forecasts.
\end{abstract}

JEL classification: Q47, D84, C33

Keywords: oil price; forecasting; internal consistency

\section{Address:}

a Christian Pierdzioch, Saarland University, Department of Economics, P.O.B. 1511 50, 66041 Saarbrücken.

${ }^{b}$ Department of Economics, WHU - Otto Beisheim School of Management, Burgplatz 2, 56179 Vallendar

${ }^{c}$ Europa-Universität Viadrina, Fakultät für Wirtschaftswissenschaften, Postfach 1786, 15207 Frankfurt (Oder) \& University of Southern Denmark

* Corresponding Author: E-mail address: jan-c.ruelke@whu.edu; Germany, Tel.: +49-261-6509-286, Fax: +49-261-6509-289.

We would like to thank the Euro Area Macroeconomic Developments Division of the European Central Bank (ECB) for providing the data. 


\title{
On the Internal Consistency of Short-Term, Medium-Term, and Long-Term Oil Price Forecasts
}

\author{
February 2011
}

\begin{abstract}
We derive internal consistency restrictions on short-term, mediumterm, and long-term oil price forecasts. We then analyze whether oil price forecasts extracted from the Survey of Professional Forecasters conducted by the European Central Bank satisfy these internal consistency restrictions. We find that neither short-term forecasts are consistent with medium-term forecasts nor that medium-term forecasts are consistent with long-term forecasts. Using a more complex expectation formation structure featuring a distributed lag structure, however, we find stronger evidence of internal consistency of mediumterm forecasts with long-term forecasts.
\end{abstract}

JEL classification: Q47, D84, C33

Keywords: oil price; forecasting; internal consistency 


\section{Introduction}

The assumption that traders in financial markets form rational forecasts of future price changes is a cornerstone of modern capital market theory. It is, therefore, not surprising that the rationality of forecasts has been tested in a large and rapidly growing empirical literature. Survey data summarizing the results of questionnaire studies of professional economists' forecasts of exchange rates, commodity prices, and macroeconomic data provide a particularly rich and reliable data source for conducting such tests (e.g., Dominguez, 1986; MacDonald and Torrance, 1988; Frankel and Froot, 1987a,b; Cavaglia et al., 1993, 1994; Menkhoff 1998, 2001; Elliott and Ito, 1999; Laster et al., 1999; Stekler, 2002). Testing whether survey data forecasts are unbiased predictors of future price changes that are orthogonal to publicly available information is a widely applied research strategy to analyze rationality of forecasts (Ito, 1990; 1993; Elliott and Ito, 1999). Testing the internal consistency of short-term, medium-term, and long-term forecasts is another research strategy. If one finds that forecasts are not consistent internally, this finding may indicate a violation of the assumption of rational forecasts (Takagi, 1991; Ito 1993; Osterberg, 2000; MacDonald, 2000).

In this paper, we studied the internal consistency of short-term, mediumterm, and long-term oil price forecasts. To this end, we derived internal consistency restrictions and tested whether oil price forecasts extracted from the Survey of Professional Forecasters conducted by the European Central Bank satisfy our internal consistency restrictions. As compared to other research strategies to test for rationality of forecasts, testing for internal consistency of forecasts at different forecasting horizons does not require forecasts to match any specific stochastic process that generates actual oil prices. In other words, rationality of forecasts requires their 
internal consistency, but internal consistency does not necessarily imply rationality of forecasts. Internal consistency of forecasts is, thus, a necessary, but not a sufficient condition for rationality of forecasts. This, perhaps, is the reason why empirical evidence of internal consistency of forecasts at different forecasting horizons is relatively scarce as compared to the voluminous research on unbiasedness and orthogonality of forecasts. Moreover, there are only a few studies using oil price forecast. The one study which uses disaggregated oil price data is by MacDonald and Marsh (1993) showing that oil price forecasts are not rational but biased.

Our empirical findings indicate that neither short-term forecasts are internally consistent with long-term forecasts nor that medium-term forecasts are internally consistent with long-term forecasts. Using a more complex expectation formation structure featuring a distributed lag structure, however, we find stronger evidence of internal consistency of medium-term forecasts with long-term forecasts. Our research builds on an early study of internal consistency of forecasts conducted by Froot and Ito (1989). Our research, however, goes beyond their study because they only analyze the internal consistency of median forecasts. In contrast, we study the internal consistency of individual forecasts in the time-series and the cross-sectional dimension of our survey data. Over and above, they analyze forecasts of exchange rates, while we study the properties of oil price forecasts. In fact, to the best of our knowledge, our study is the first empirical study focusing on the internal consistency of oil price forecasts. Our empirical study is also among the first studies that analyze data from the Survey of Professional Forecasters because the European Central Bank has released this data only recently.

We organize the remainder of this paper as follows. In Section 2, we describe our survey data. In Section 3, we test for internal consistency of 
forecasts by assuming a distributed-lag model featuring one lag to capture how forecasters form their oil price forecasts. In Section 4, we extend the distributed-lag model to include a more complex lag structure. In Section 5, we conclude.

\section{Survey Data of Oil Price Forecasts}

In order to study the internal consistency of oil price forecasts, we extracted oil price forecasts from the Survey of Professional Forecasters (SPF), which is conducted and compiled by the European Central Bank (ECB). The forecasters who participate in the survey work for investment banks, large international corporations, economic research institutes, and at universities. Our sample period covers the period of time from 2002Q1 to 2009Q4. Because the ECB publishes the SPF forecasts at a quarterly frequency, we could analyze survey data from of 32 forecasting cycles.

In total, 88 forecasters participated in the survey, where we limited our analysis to those forecasters who participated in all surveys. This applies to 25 forecasters and yields a balanced data set. The SPF data, thus, contain oil price forecasts of a substantial number of individual forecasters, and not only the cross-sectional mean or median of a group of forecasters. This rich cross-sectional dimension of the SPF data renders it possible to study both the time-series dimension and the cross-sectional characteristics of the SPF data by applying econometric techniques developed for the analysis of panel data.

At the beginning of a quarter, the ECB asks the forecasters about their projection of the oil price for various time horizons. As a consequence, we can analyze forecasts for three different forecast horizons: one-quarter-ahead 
oil price forecasts, two-quarters-ahead oil price forecasts, and four-quartersahead oil price forecasts. In our empirical analysis, we refer to these forecasts as short-term, medium-term, and long-term oil price forecasts. In total, we had available 800 oil price forecasts. Figures $1-3$ plot the oil price forecasts at the three available forecasting horizons. The figures plot the actual oil price (solid lines), the cross-sectional average of oil prices (dashed lines), and the cross-sectional range of price forecasts (shaded areas). The latter is defined as the difference between the maximum and the minimum of oil price forecasts per forecasting cycle.

- Insert Figures 1, 2, and 3 about here.

The figures illustrate that the price of oil fluctuated widely during the sample period under consideration. Our sample period also covers the recent oil price roller coaster of 2008 following the U.S. subprime mortgage crisis. Eyeballing the figures further shows that the cross-sectional average of oil price forecasts persistently deviated from the actual oil price, suggesting that oil price forecasts may be biased. The result that SPF oil price forecasts are biased is consistent with the results reported by MacDonald and Marsh (1993), who find that the oil price forecasts published in the Consensus Economic Forecast poll are biased and inefficient. Their analysis, however, is limited to the period of time from October 1989 to March 1991, which is a relatively short period of time compared to our sample period of eight years.

Figures 1-3 show that the width of the cross-sectional range of price forecasts is substantial indicating a significant amount of dispersion and heterogeneity in oil price forecasts. In other words, it is interesting to analyze both the time-series dimension and the cross-sectional dimension of the SFP oil price forecasts as the latter is likely to yield important insights into how forecasters form oil price forecasts. It is also evident from the figures that the cross-sectional range of oil price forecasts increases as one 
moves from the analysis of short-term forecasts in Figure 1 to the analysis of long-term-forecasts in Figure 3. While it seems that the cross-sectional averages of short-term, medium-term, and long-term oil price forecasts move in tandem, a formal econometric analysis that takes into account the cross-sectional dimension of the SPF survey data is needed to decide whether the SPF oil price forecasts are internally consistent at different forecast horizons.

\section{Forecast Consistency in the Baseline Model}

We analyzed the internal consistency of the SPF oil price forecasts at different forecasting horizons by deriving consistency restrictions similar to those analyzed by Froot and Ito (1989) in their analysis of exchange rate forecasts. According to their definition, internal consistency of forecasts requires that forecasts delivered in the same forecasting cycle, but measured at different forecasting horizons yield the same result. Expressed more formally, two forecasts are said to be internally consistent if a short-term forecast, $E_{t}\left(s_{t+k}\right)$, formed in period $t$ of the oil price in period $t+k$ is identical to a long-term forecast, $E_{t}\left(s_{t+k+j}\right)$, formed in period $t$ of the oil price in period $t+k+j$, when the model that describes the dynamics of the short-term forecast is iterated $j>0$ periods forward in time.

This definition of internal consistency of oil price forecasts implies empirically testable hypotheses that come in the form of cross-equation constraints on coefficients of short-term and longer-term forecasting equations. In order to setup such forecasting equations, we followed Frankel and Froot (1987a,b) and assumed that oil price forecasters derive their forecasts from an extrapolative forecasting equation. Such an extrapolative forecasting equation can 
be expressed, in its simplest form, as a distributed-lag model as follows:

$$
E_{t ; i}\left[s_{t+k}\right]-s_{t}=\alpha_{k}+\beta_{k}\left(s_{t-1}-s_{t}\right)+\epsilon_{t ; i}
$$

where $s_{t}$ denotes the oil price in period $t$, and $E_{t}\left[s_{t+k}\right]$ denotes the log of the oil price forecast for period $t+k$ delivered by forecaster $i$ at time $t$. The subscript, $k$, denotes the forecast horizon and $\epsilon_{t}$ denotes a stochastic error term. We define the change in the oil price as $s_{t-1}-s_{t}$ rather than as $s_{t}-s_{t-1}$ in order to derive the consistency restrictions in a mathematically convenient way. In economic terms, a negative coefficient, $\beta_{k}$, indicates that, whenever the oil price increases, forecasters expect a further increase in the price of oil. This is referred to as bandwagon expectations. In contrast, forecasters form contrarian expectations if the coefficient, $\beta_{k}$, is positive. In this case, forecasters predict a decrease in the oil price whenever the price oil price increased just before a new forecasting cycle starts.

Upon applying Equation (1) to the short-term, medium-term, and long-term forecasts, we obtain

$$
\begin{gathered}
E_{t ; i}\left[s_{t+1}\right]-s_{t}=\alpha_{1}+\beta_{1}\left(s_{t-1}-s_{t}\right)+\epsilon_{t}, \\
E_{t ; i}\left[s_{t+k}\right]-s_{t}=\alpha_{2}+\beta_{2}\left(s_{t-1}-s_{t}\right)+u_{t}, \quad k=2,4 .
\end{gathered}
$$

It should be noted from Equations (2) and (3) that the time frequency is different when we compare short-term forecasts to medium-term forecasts and medium-term forecasts to long-term forecasts. In the case of the former comparison, one period refers to one quarter while in the case of the latter comparison, one period refers to two quarters. Hence, we estimated Equation (1) for the medium-term forecasts twice using different time scales so as to generate two sets of coefficients that can be used for the two different comparisons. 
Internal consistency of forecasts at different forecasting horizons requires that iteration of the model of short-term forecasts given in Equation (2) yields the same forecast at a longer forecasting horizon as the model of longterm forecasts specified in Equation (3). Internally consistent forecasts, thus, must satisfy the following two internal consistency restrictions (Appendix A):

$$
\begin{aligned}
& \alpha_{2}=2 \alpha_{1}-\beta_{1} \alpha_{1} \\
& \beta_{2}=\beta_{1}\left(1-\beta_{1}\right) .
\end{aligned}
$$

The economic interpretation of the internal consistency restrictions, $R 1$ and $R 2$, is straightforward. For example, the internal consistency restriction $R 1$ requires that, if a forecaster forecasts that oil prices increase by $\alpha_{1}$ in the short term, then the forecaster should forecast an increase in the oil price of $2 \alpha_{1}$ in the medium-term, correcting for the intermediate change in the price of oil, $\beta_{1} \alpha_{1}$, that is being the short-term forecast. As regards the internal consistency restriction $R 2$, a similar logic applies. Neglecting the intercept terms, $\alpha_{1}$ and $\alpha_{2}$, the distributed-lag model implies that a forecast is the weighted sum of the current and the past oil price, where the short-term weighting factors are given by $1-\beta_{1}$ and $\beta_{1}$. It follows that, if a forecaster forecasts that, in the short-term, the past price of oil transmits onto the next period oil price by an amount of $\beta_{1}$, then an amount of $1-\beta_{1}$ is left for adjustment two periods ahead. For forecasts to be internally consistent, it must thus be the case that the forecast of the total medium-term adjustment, $\beta_{2}$, is linked to the forecast of the short-term adjustment via internal consistency restriction $R 2$. In addition, the internal consistency restriction, $R 2$, reveals that as long as the short-term $\beta$-coefficients assume values between -1 and 1 (as they do in our empirical analysis), the $\beta$-coefficients must have the same sign across forecast horizons for internal consistency to hold. Put differently, a forecaster who delivers contrarian (bandwagon) forecasts in the short-term must also deliver contrarian (bandwagon) forecasts 
at longer forecasting horizons to meet the internal consistency restriction $R 2$.

We estimated Equations (2) and (3) on the SPF survey data of short-term forecasts, medium-term forecasts, and long-term forecasts of the price of oil. Table 1 summarizes the estimation results which are based on the heteroskedasticity and serial correlation-consistent Newey-West panel estimator (Newey and West, 1987). The positive $\beta$-coefficients for all forecast horizons indicate that oil price forecasters form contrarian expectations. For example, the coefficient of 0.06 for the one-quarter-ahead forecast reflects that forecasters expect the oil price to increase by 0.06 per cent if the oil price decreased by one percent in the preceding quarter. The $\beta$-coefficients for the longer-term forecasts are larger than the coefficient for the shorter-term forecasts indicating perceived mean-reversion in the price of oil as the forecast horizon increases.

- Insert Table 1 about here.

With regard to the internal consistency restrictions, Table 2 indicates that internal consistency restriction $R 1$ can be rejected at the one percent level of significance for all forecast horizons, while internal consistency restriction $R 2$ can be rejected at the one (five) percent level of significance in the case of medium-term versus long-term forecasts (short-term versus medium-term forecasts). Additionally, both internal consistency restrictions, $R 1$ and $R 2$, can be rejected jointly at the one percent level.

- Insert Table 2 about here. -

So far, we restricted $\beta_{2}$ to be common across all professional forecasters. Given the evidence of cross-sectional heterogeneity of forecasts (Figures $1-3)$, we refined our analysis by analyzing the link between shortterm, medium-term and long-term $\beta$-coefficients of individual forecasters. To 
this end, we computed, in a first step, short-term, medium-term and longterm $\beta$-coefficients by estimating versions of Equation (2) and (3) featuring forecaster-specific $\beta$-coefficients. We used, in a second step, the estimated forecaster-specific $\beta$-coefficients to analyze a potential non-linear link between the forecaster-specific $\beta$-coefficients at different forecasting horizons. To this end, we considered the following equation:

$$
\beta_{i, 2}=\alpha+\psi_{1} \beta_{i, 1}+\psi_{2} \beta_{i, 1}^{2}+\omega_{i}
$$

where $i$ denotes a forecaster index and $\omega_{i}$ denotes an error term. Internal consistency of forecasts across the different forecasting horizons requires according to consistency restriction $(R 2)$ - a constant term of $\alpha=0$, and a slope parameter of $\psi_{1}=1$ and a concavity parameter of $\psi_{2}=-1$. Table 3 summarizes the estimation results for Equation (4). While it is possible to reject the null hypothesis that the oil price forecasts are consistent with internal consistency restriction $R 2$, only the null hypothesis $\alpha=0$ can be rejected in the case of medium-term versus long-term forecasts. The other parameters of Equation (4) are in line with the internal consistency restriction $R 2$. In particular, the parameter $\psi_{1}$ is not different from unity for both specifications which is in line with the internal consistency restriction $R 2$.

- Insert Table 3 about here. -

Figures 4-5 illustrate the estimation results for Equation (4). The figures illustrate the link between the forecaster-specific short-term, medium-term and long-term $\beta$-coefficients, which are shown as dots in the figures. The solid line represents the estimation results obtained from Equation (4) and the dotted line represents the internal consistency restriction, $R 2$. As evidenced by Figures 4-5, there is a positive relationship between the forecaster-specific short-term, medium-term, and long-term $\beta$-coefficients, which reflects the significantly positive $\psi_{1}$ coefficients in Table 3 . Hence, a forecaster who attached a substantial weight to past oil price developments in order to extrapolate the 
short-term oil price is likely to do the same when forming medium-term and long-term forecasts. The $\beta$-coefficients, however, differ significantly across forecasters at all forecast horizons.

- Insert Figures 4, and 5 about here.

Combinations of the $\beta$-coefficients in the upper left and lower right quadrants of Figures 4 and 5 reflect that a few forecasters have negative (positive) short-term (medium-term) $\beta$-coefficients and, at the same time, positive (negative) medium-term (long-term) $\beta$-coefficients. These forecasters exhibit what has been referred to in the earlier literature as twisting behavior. While this feature is well established regarding exchange-rate forecasts (Ito, 1990), our empirical findings suggest that twisting behavior is not very pronounced in oil price forecasts. We only find four (one) forecasters with such a behavior when comparing the $\beta$-coefficients of the short-term (medium-term) forecasts with those of the (long-term) forecasts.

\section{Forecast Consistency in an Extended Model}

As an extension of our empirical analysis and to analyze the robustness of our results, we considered a distributed-lag model featuring two lags to model the process of forecast formation. It is also worth mentioning that the dynamics implied by such an extended distributed-lag model are rich enough to allow for twisting behavior of internally consistent forecasts. In other words, the extended distributed-lag model renders it possible to model an internally consistent twist of short-term bandwagon and long-term contrarian forecasts. We considered the following extended distributed-lag model:

$$
\begin{aligned}
& E_{t}\left[s_{t+1}\right]-s_{t}=\alpha_{1}+\beta_{1} s_{t}+\gamma_{1} s_{t-1}+\delta_{1} s_{t-2}+\phi_{t} . \\
& E_{t}\left[s_{t+2}\right]-s_{t}=\alpha_{2}+\beta_{2} s_{t}+\gamma_{2} s_{t-1}+\delta_{2} s_{t-2}+\chi_{t},
\end{aligned}
$$


where $\phi_{t}$ and $\chi_{t}$ are error terms. As shown at the end of the paper (Appendix B), internally consistent long-term forecasts must satisfy the following internal consistency restrictions:

$$
\begin{aligned}
& \alpha_{2}=2 \alpha_{1}+\alpha_{1} \beta_{1} \\
& \beta_{2}=2 \beta_{1}+\beta_{1}^{2}+\gamma_{1} \\
& \gamma_{2}=\beta_{1} \gamma_{1}+\gamma_{1}+\delta_{1} \\
& \delta_{2}=\delta_{1}+\beta_{1} \delta_{1}
\end{aligned}
$$

Internal consistency restriction $R 5$ shows that a distributed-lag model featuring two lags implies that short-term forecasts can be consistent with contrarian long-term forecasts even in case of a negative short-term $\beta$-coefficient. In other words, the internal consistency restriction $R 4$ may be satisfied even if a forecaster forms bandwagon short-term forecasts that come along with contrarian long-term forecasts.

- Insert Table 4 and 5 about here.

Table 4 presents the estimation results for Equations (5) and (6) based on the Newey-West panel estimator. The estimation results reveal that, as in Section 3 , forecasters predict on average a decrease in the oil price, as reflected by the significantly negative constant term. Interestingly, only the $\delta$ coefficient is significant in all specifications. As the results summarized in Table 5 indicate, the joint internal consistency restrictions can be soundly rejected at all forecast horizons, implying that short-term, medium-term, and longterm forecasts of the oil price are internally inconsistent even if one allows for two lags in the distributed-lag model. In the case of the comparison of the medium-term with the long-term forecasts, however, the evidence of internal inconsistency of forecasts is not overwhelmingly strong because the internal consistency restrictions $R 4, R 5$, and $R 6$ cannot be rejected. It is the internal 
consistency restriction $R 3$, which includes the $\alpha$-coefficients and, thus, any systematic biases in oil price forecasts, that is responsible for the rejection of internal consistency of forecasts.

\section{Conclusion}

We have derived and applied internal consistency restrictions to study the internal consistency of short-term, medium-term, and long-term forecasts of the price of oil. To this end, we used the Survey of Professional Forecaster provided by the European Central Bank. Neither short-term forecasts are consistent with medium-term forecasts nor medium-term forecasts are consistent with long-term forecasts. Considering a more complex forecast formation model, however, we have found stronger evidence of internal consistency of medium-term forecasts with long-term forecasts.

Our empirical results indicate that an interesting avenue for future research is to describe oil price dynamics in terms of models that do not rely on the assumption that market participants, traders, and forecasters form rational expectations. Our empirical results indicate that models featuring heterogeneous groups of traders, like chartists and fundamentalists (Reitz and Slopek, 2009) may turn out to be particularly useful in this respect. Various heterogeneous-traders models have been developed, for example, to model the dynamics of exchange rates (De Grauwe and Grimaldi, 2006).

When applying such models to the modeling of oil price dynamics, one has to account for potentially important structural differences between the oil market and the market for foreign currency. For example, our empirical results indicate that twisting behavior seems to be less much prevalent in the oil market than in foreign-exchange markets. It may also turn out that the specific structural characteristics of the oil market require changes in 
standard calibrations of heterogeneous-trader models. Our empirical results may also be useful to adapt calibrations of heterogeneous-trader models to the modeling of oil price dynamics. 


\section{References}

Cavaglia S, Verschoor WFC, Wolff CCP. 1993. Further Evidence on Exchange Rate Expectations. Journal of International Money and Finance $12,78-98$.

Cavaglia S, Verschoor WFC, Wolff CCP. 1994. On the Biasedness of Forward Foreign Exchange Rates: Irrationality or Risk Premia? Journal of Business 67, 321-343.

De Grauwe P, Grimaldi M. 2006. Exchange Rate Puzzles: A Tale of Switching Attractors, European Economic Review 50, 1-33.

Dominguez KM. 1986. Are Foreign Exchange Forecasts Rational? New Evidence from Survey Data. Economics Letters 21, 277-281.

Elliott G, Ito T. 1999. Heterogeneous Expectations and Tests of Efficiency in the Yen/Dollar Forward Exchange Rate Market. Journal of Monetary Economics 43, 435-454.

Frankel JA, Froot KA. 1987a. Using Survey Data to Test Standard Propositions Regarding Exchange Rate Expectations. American Economic Review 77, 133-153.

Frankel JA, Froot KA. 1987b, Short-Term and Long-Term Expectations of the Yen/Dollar Exchange Rate: Evidence from Survey Data. Journal of the Japanese and International Economy 1, 249-274.

Froot KA, Ito T. 1989. On the Consistency of Short-Run and Long-Run Exchange Rate Expectations. Journal of International Money and Finance 8, 487-510.

Ito T. 1990. Foreign Exchange Rate Expectations: Micro Survey Data. The American Economic Review 80, 434-449.

Ito T. 1993. Foreign Exchange Rate Expectations: Micro Survey Data. Das DK, ed. International Finance: Contemporary Issues Routledge: 360-374.

Laster D, Bennett P, Geoum IS. 1999. Rational Bias in Macroeconomic Forecasts. The Quarterly Journal of Economics 114, 293-318.

MacDonald R. 2000. Expectation Formation and Risk in Three Financial Markets: Surveying What The Survey Says. Journal of Economic Surveys $14,69-100$. 
MacDonald R, Marsh IW. 1993. On the Efficiency of Oil Price Forecasts, Applied Financial Economics 3, 293-302.

MacDonald R, Torrance TS. 1988. On Risk, Rationality and excessive Speculation in the Deutschmark-US Dollar Exchange Market: Some Evidence using Survey Data. Oxford Bulletin of Economics and Statistics 50, 107-123.

Menkhoff L. 1998. The Noise Trading Approach - Questionnaire Evidence from Foreign Exchange. Journal of International Money and Finance $17,547-564$.

Menkhoff L. 2001. Short-Term Horizons in Foreign Exchange? Survey Evidence from Dealers and Fund Managers. Kyklos 54, 27-48.

Newey W, West KD. 1987. A Simple, Positive Semi-Definite, Heteroskedasticity and Autocorrelation Consistent Covariance Matrix, Econometrica 55, 703-708.

Osterberg WP. 2000. New Results on the Rationality of Survey Measures of Exchange Rate Expectations. Federal Reserve Bank of Cleveland Economic Review 36, 14-21.

Reitz S, Slopek U. 2009. Nonlinear Oil Price Dynamics - A Tale of Heterogeneous Speculators?, German Economic Review 10, 270-283.

Stekler HO. 2002. The Rationality and Efficiency of Individuals Forecasts. Clements MP, Hendry DF, ed.A Companion to Economic Forecasting. Oxford: Blackwells; 222-240.

Takagi, S. 1991. Exchange Rate Expectations, a Survey of Survey Studies. IMF Staff Papers 38, 156-183. 


\section{Appendix A: Internal Consistency Restrictions for a Distributed-Lag Model With One Lag}

Starting from

$$
\begin{aligned}
& E_{t}\left[s_{t+1}\right]-s_{t}=\alpha_{1}+\beta_{1}\left(s_{t-1}-s_{t}\right)+\epsilon_{t}, \\
& E_{t}\left[s_{t+2}\right]-s_{t}=\alpha_{2}+\beta_{2}\left(s_{t-1}-s_{t}\right)+u_{t},
\end{aligned}
$$

and after rearranging terms, one obtains

$$
\begin{aligned}
& E_{t}\left[s_{t+1}\right]=\alpha_{1}+\left(1-\beta_{1}\right) s_{t}+\beta_{1} s_{t-1}+\epsilon_{t}, \\
& E_{t}\left[s_{t+2}\right]=\alpha_{2}+\left(1-\beta_{2}\right) s_{t}+\beta_{2} s_{t-1}+u_{t} .
\end{aligned}
$$

Upon iterating Equation (A1) forward in time, and applying the law of iterated exceptions, a new equation for long-term forecasts can be obtained:

$$
E_{t}\left[s_{t+2}\right]=\alpha_{1}+\left(1-\beta_{1}\right) E_{t}\left[s_{t+1}\right]+\beta_{1} s_{t} .
$$

Upon subtracting $s_{t}$ from both sides of Equation (A5), and using Equation (A1), one can derive

$$
E_{t}\left[s_{t+2}\right]-s_{t}=2 \alpha_{1}-\beta_{1} \alpha_{1}+\left(1-\beta_{1}\right) \beta\left(s_{t-1}-s_{t}\right)+\left(1-\beta_{1} \epsilon_{t}\right) .
$$

A comparison of Equation (A8) with Equation (A2) yields the internal consistency restrictions (R1) and (R2). 


\section{Appendix B: Internal Consistency Restrictions for a Distributed-Lag Model With Two Lags}

Starting from

$$
\begin{aligned}
& E_{t}\left[s_{t+1}\right]-s_{t}=\alpha_{1}+\beta_{1} s_{t}+\gamma_{1} s_{t-1}+\delta_{1} s_{t-2}+\phi_{t}, \\
& E_{t}\left[s_{t+2}\right]-s_{t}=\alpha_{2}+\beta_{2} s_{t}+\gamma_{2} s_{t-1}+\delta_{2} s_{t-2}+\chi_{t},
\end{aligned}
$$

rearranging terms yields

$$
\begin{aligned}
& E_{t}\left[s_{t+1}\right]=\alpha_{1}+\left(1+\beta_{1}\right) s_{t}+\gamma_{1} s_{t-1}+\delta_{1} s_{t-2}+\phi_{t}, \\
& E_{t}\left[s_{t+2}\right]=\alpha_{2}+\left(1+\beta_{2}\right) s_{t}+\gamma_{2} s_{t-1}+\delta_{2} s_{t-2}+\chi_{t} .
\end{aligned}
$$

From iterating Equation (B3) one period forward in time, and applying the law of iterated expectations, it follows that

$$
E_{t}\left[s_{t+2}\right]=\alpha_{1}+\left(1+\beta_{1}\right) E_{t}\left[s_{t+1}\right]+\gamma_{1} s_{t}+\delta_{1} s_{t-1} .
$$

Upon subtracting $s_{t}$ from both sides of Equation (B5), using Equation (B1), and equating the resulting coefficients with the coefficients in Equation (B2) yields the internal consistency restrictions (R3)-(R6). 


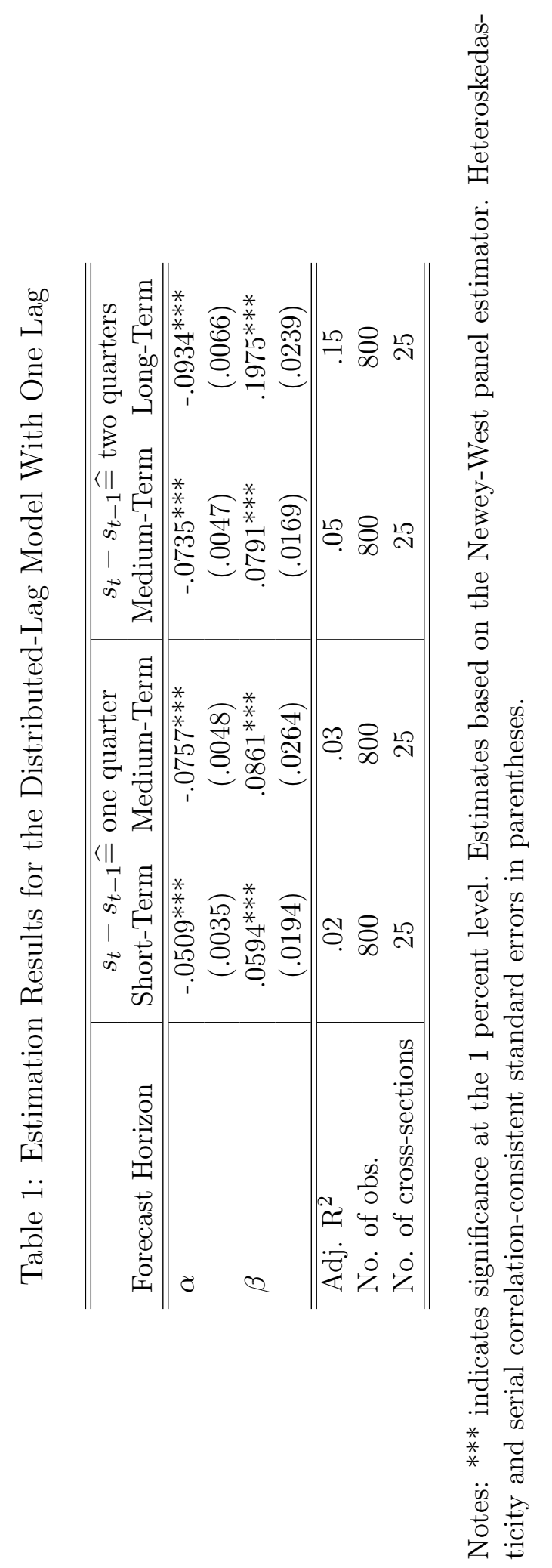


Table 2: Test of Internal Consistency Restrictions $R 1$ and $R 2$

\begin{tabular}{l|c|c}
\hline \hline Forecast Horizon & Short- vs. Medium-Term & Medium- vs. Long-Term \\
\hline \hline$R 1$ & $27.07^{* * *}$ & $77.94^{* * *}$ \\
& $(.00)$ & $(.00)$ \\
$R 2$ & $3.86^{* *}$ & $89.53^{* * *}$ \\
& $(.05)$ & $(.00)$ \\
\multirow{2}{*}{ and $R 2$} & $29.37^{* * *}$ & $164.17^{* * *}$ \\
& $(.00)$ & $(.00)$ \\
\hline \hline
\end{tabular}

Notes: The table summarizes F-values. The null hypothesis is that the internal consistency restrictions hold. ${ }^{* * *}(* *)$ denote significance at the $1(5)$ percent level. Significance levels are given in parentheses.

Table 3: Equation Linking Short-Term and Long-Term $\beta$-Coefficients

\begin{tabular}{l|c|c}
\hline \hline Forecast Horizon & Short- vs. Medium-Term & Medium- vs. Long-Term \\
\hline \hline$\alpha$ & .0187 & $.0982^{* * *}$ \\
& $(.0147)$ & $(.0191)$ \\
$\psi_{1}$ & $1.0904^{* * *}$ & $1.3831^{* * *}$ \\
& $(.1981)$ & $(.2779)$ \\
$\psi_{2}$ & .2148 & -.7463 \\
& $(.9885)$ & $(1.2377)$ \\
\hline \hline Adj. $\mathrm{R}^{2}$ & .68 & .72 \\
$H_{0}: \alpha=0$ & .32 & .00 \\
$H_{1}: \psi_{1}=1$ & .72 & .18 \\
$H_{2}: \psi_{2}=-1$ & .23 & .84 \\
$H_{0} \& H_{1} \& H_{2}$ & .02 & .00 \\
No. of obs. & 25 & 25 \\
\hline \hline
\end{tabular}

Notes: Estimated equation: $\beta_{i, 2}=\alpha+\psi_{1} \beta_{i, 1}+\psi_{2} \beta_{i, 1}^{2}+\omega_{i}$, for $i=1, \ldots, 25$. $* * *$ indicates significance at the 1 percent level. Estimates based on the NeweyWest panel estimator. Heteroskedasticity and serial correlation-consistent standard errors in parentheses. 


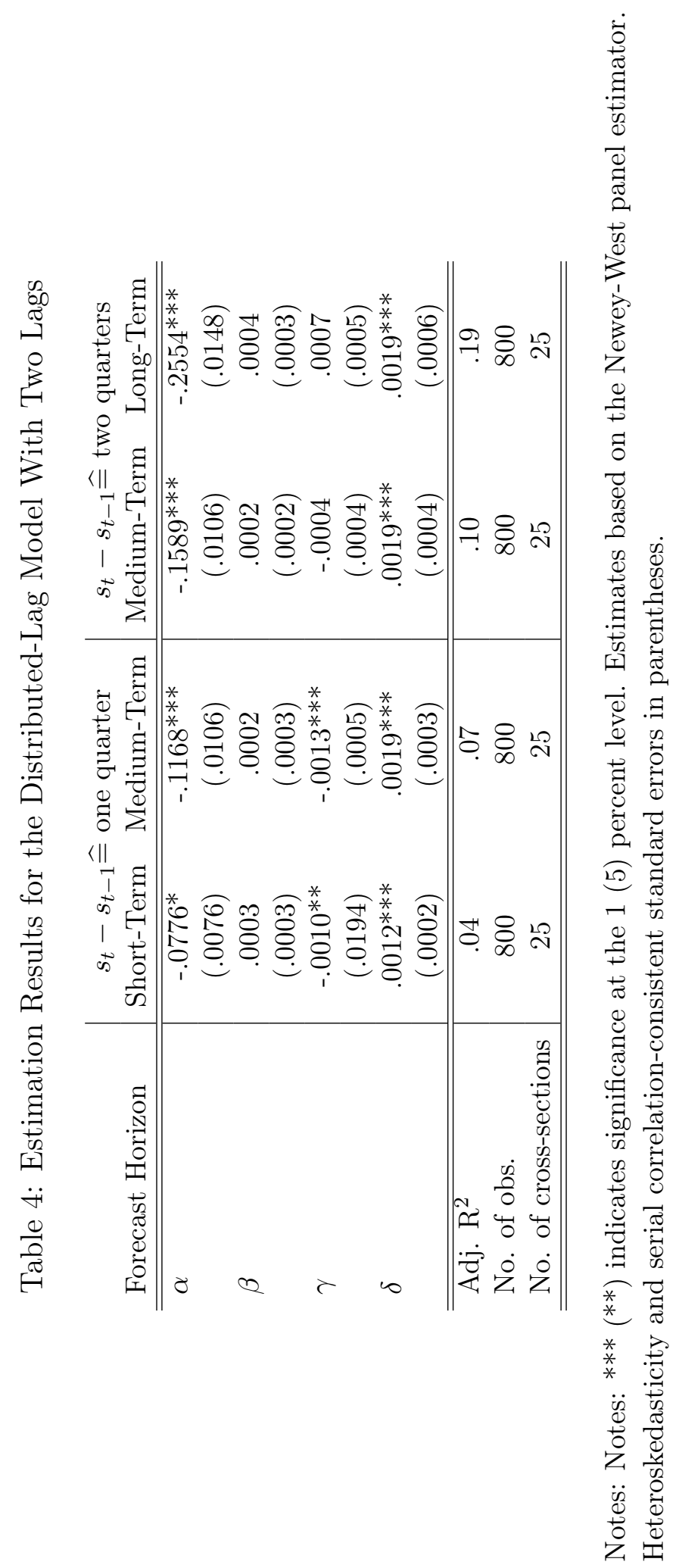


Table 5: Test of Internal Consistency Restrictions $R 3, R 4, R 5$, and $R 6$

\begin{tabular}{l|c|c}
\hline \hline Period & Short- vs. Medium-Term & Medium- vs. Long-Term \\
\hline \hline$R 3$ & $14.94^{* * *}$ & $22.34^{* * *}$ \\
& $(.00)$ & $(.00)$ \\
$R 4$ & 2.67 & 1.03 \\
& $(.10)$ & $(.31)$ \\
$R 5$ & $18.79^{* * *}$ & 2.12 \\
& $(.00)$ & $(.15)$ \\
$R 6$ & $14.61^{* * *}$ & 0.03 \\
& $(.00)$ & $(.86)$ \\
$R 3$ to $R 6$ & $72.47^{* * *}$ & $82.70^{* * *}$ \\
& $(.00)$ & $(.00)$ \\
\hline \hline
\end{tabular}

Notes: The table summarizes F-values. The null hypothesis is that the internal consistency restrictions hold. ${ }^{* * *}(* *)$ denote significance at the 1 (5) percent level. Significance levels are given in parentheses.

Figure 1: One-Quarter-Ahead Forecast and Actual Oil Price

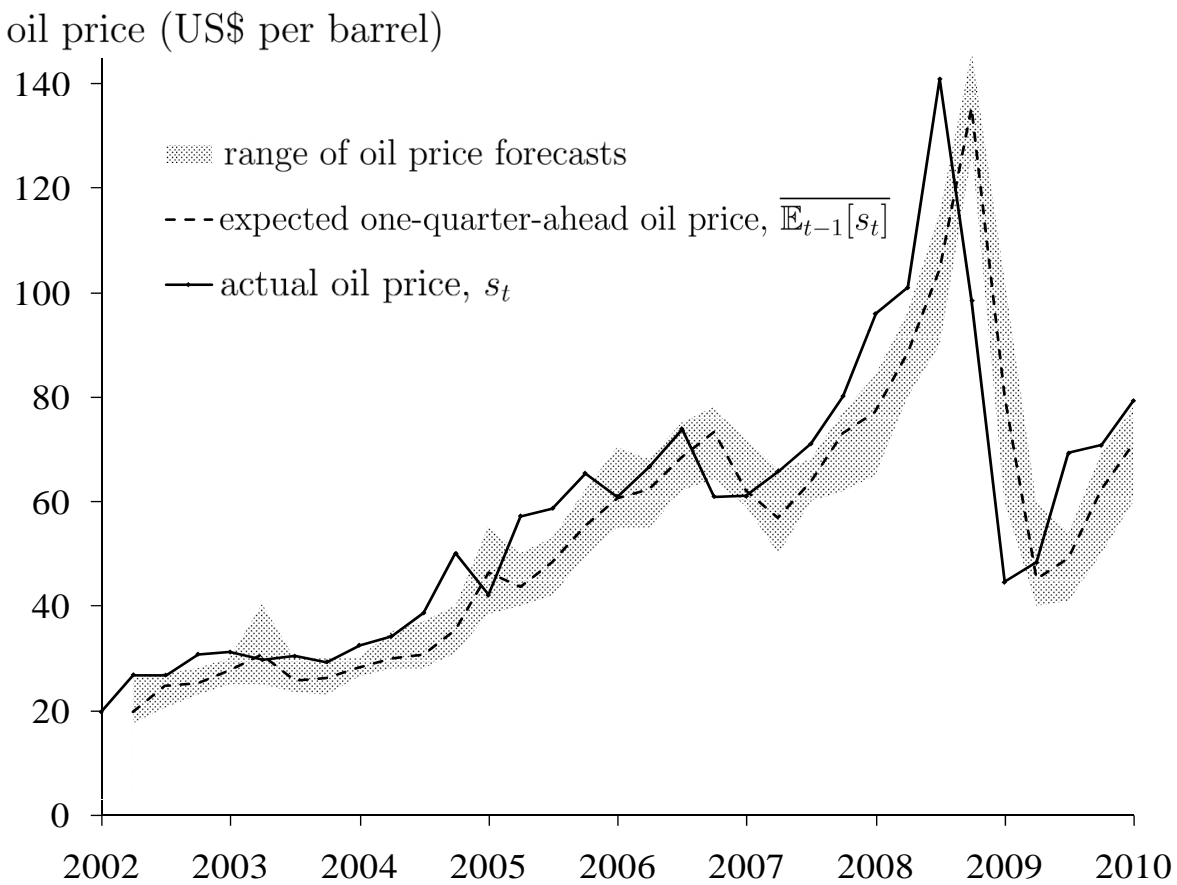


Figure 2: Two-Quarters-Ahead Forecast and Actual Oil Price

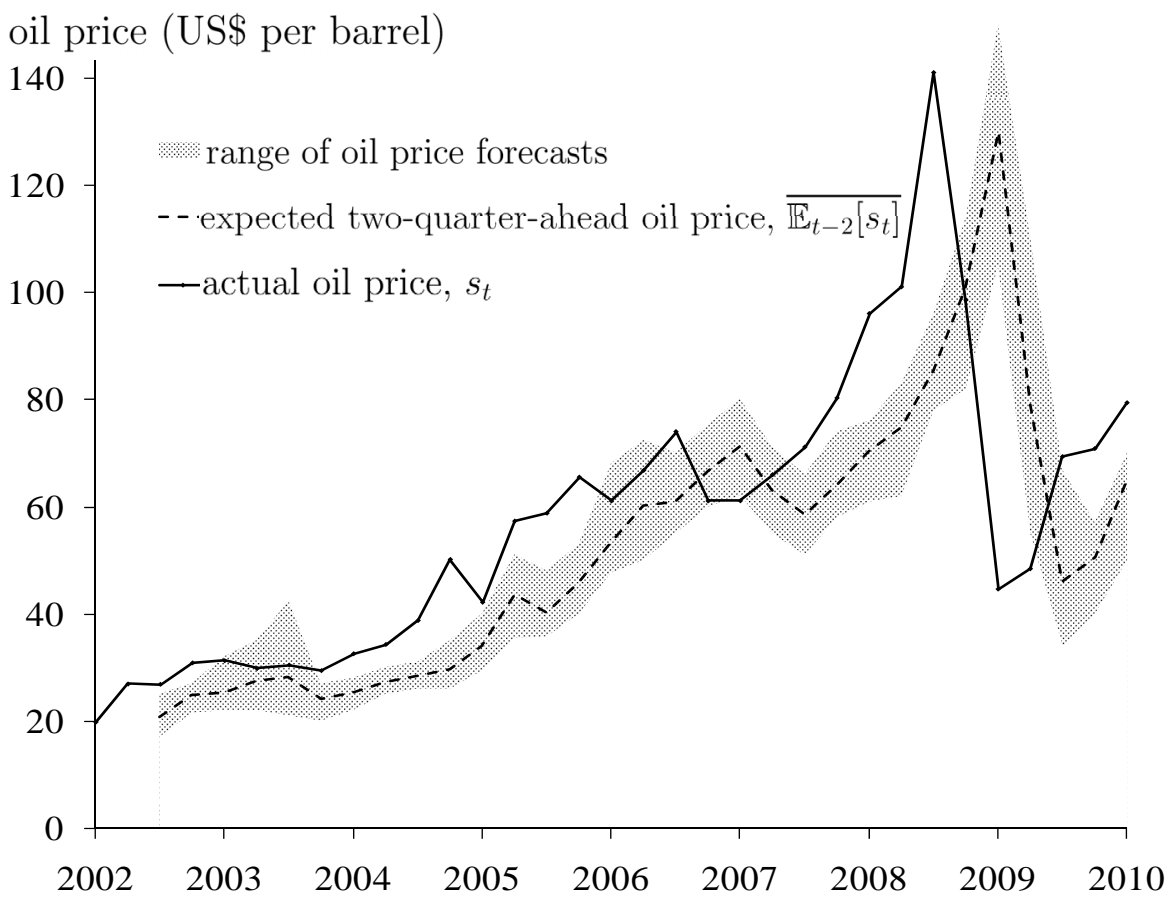

Figure 3: Four-Quarters-Ahead Forecast and Actual Oil Price

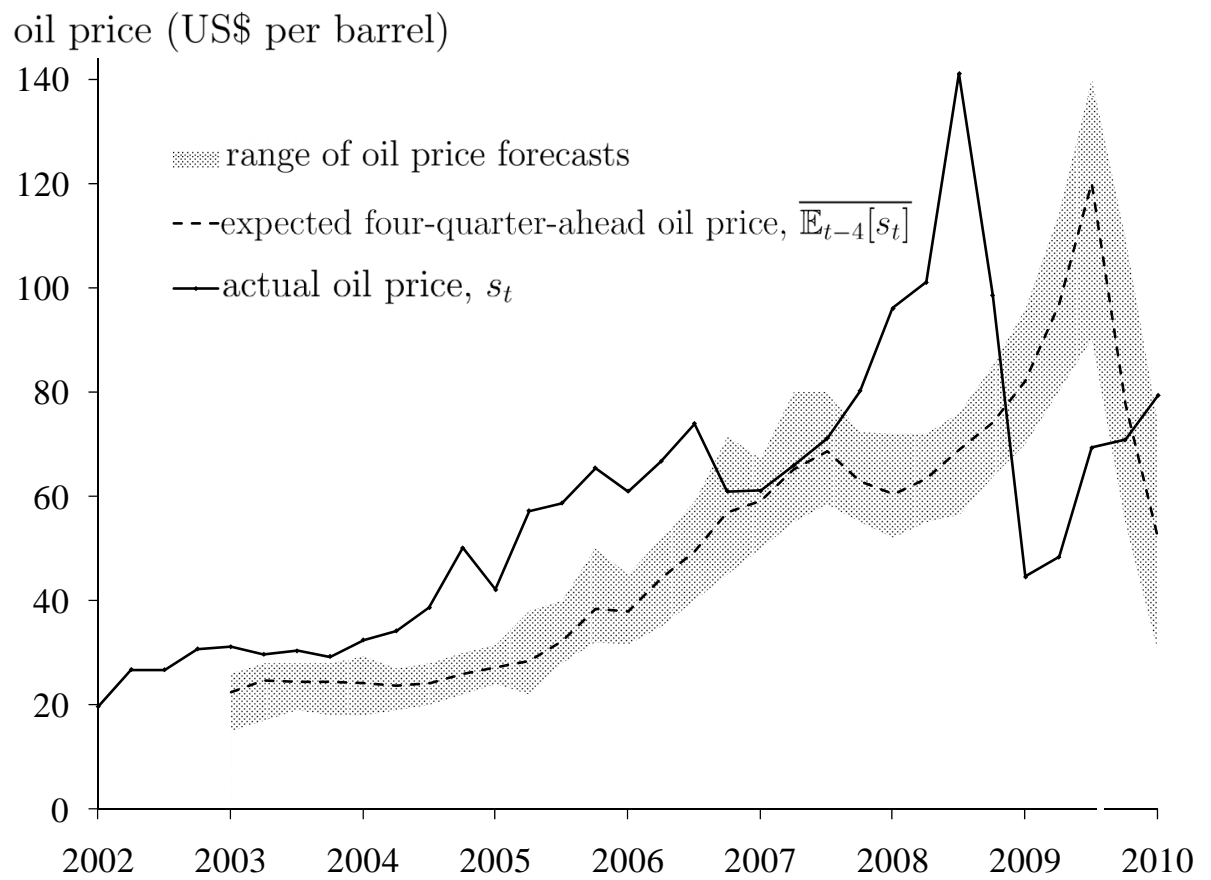


Figure 4: Individual $\beta$-Coefficients for Short-Term and Medium-Term Oil Price Forecasts

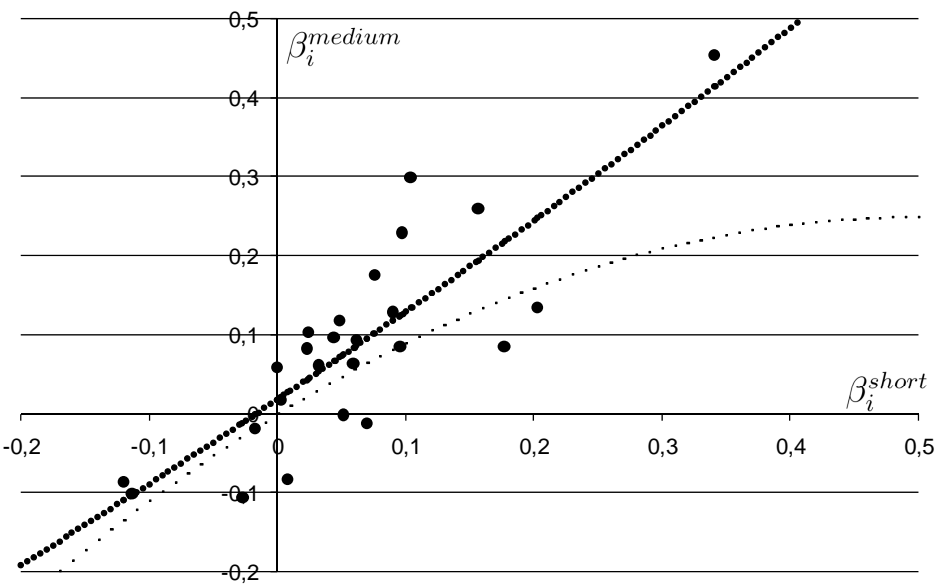

Note: The dots denote the individual $\beta^{\text {short }}$ and $\beta^{\text {medium }}$ coefficients. The dotted line represents consistency restriction $(R 2)$. The solid line represents the estimated non-linear regression model (Equation $(4))$.

Figure 5: Individual $\beta$-Coefficients for Medium-Term and Long-Term Oil Price Forecasts

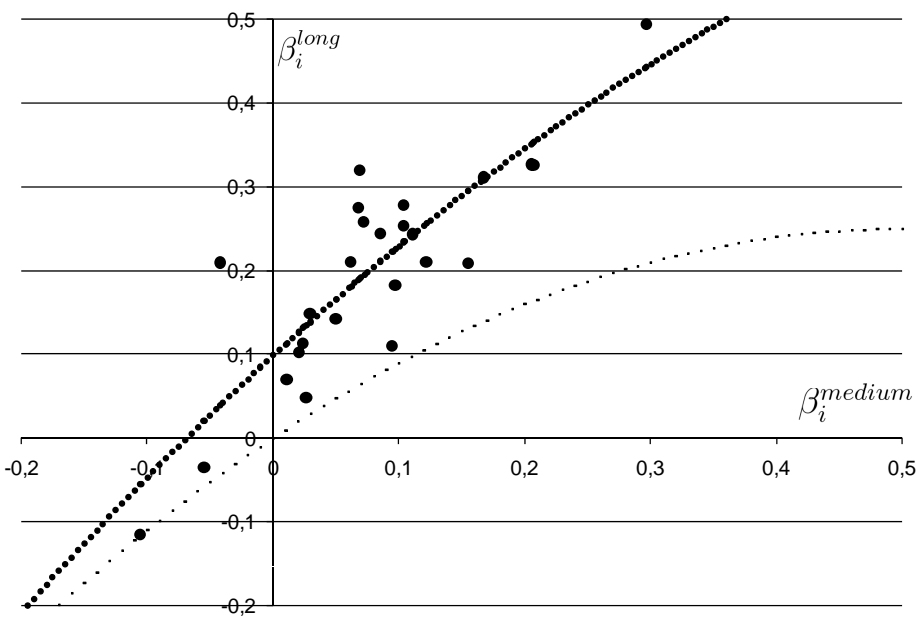

Note: The dots denote the individual $\beta^{\text {medium }}$ and $\beta^{\text {long }}$ coefficients. The dotted line represents consistency restriction $(R 2)$. The solid line represents the estimated non-linear regression model (Equation $(4))$. 